\title{
Oryzias curvinotus in Sanya Does Not Contain the Male Sex-Determining Gene dmy
}

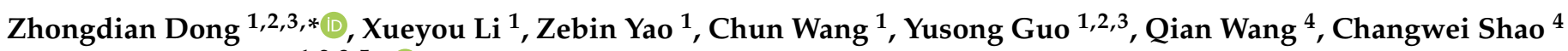 \\ and Zhongduo Wang $1,2,3,5, *$ (1)
}

1 Guangdong South China Sea Key Laboratory of Aquaculture for Aquatic Economic Animals, Fisheries College, Guangdong Ocean University, Zhanjiang 524025, China; 1xycjhj@163.com (X.L.); yaozebin@stu.gdou.edu.cn (Z.Y.); chunwang_2019@163.com (C.W.); ysguo@gdou.edu.cn (Y.G.)

2 Guangdong Provincial Engineering Laboratory for Mariculture Organism Breeding, Fisheries College, Guangdong Ocean University, Zhanjiang 524025, China

3 Guangdong Provincial Key Laboratory of Pathogenic Biology and Epidemiology for Aquatic Economic Animals, Fisheries College, Guangdong Ocean University, Zhanjiang 524025, China

4 Yellow Sea Fisheries Research Institute, Chinese Academy of Fisheries Sciences, Qingdao 266071, China; wangqian2014@ysfri.ac.cn (Q.W.); shaochangwei303@163.com (C.S.)

5 State Laboratory of Developmental Biology of Freshwater Fish, College of Life Sciences, Hunan Normal University School, Changsha 410081, China

* Correspondence: zddong@gdou.edu.cn (Z.D.); aduofa@hotmail.com (Z.W.)

Citation: Dong, Z.; Li, X.; Yao, Z.; Wang, C.; Guo, Y.; Wang, Q.; Shao, C.; Wang, Z. Oryzias curvinotus in Sanya Does Not Contain the Male Sex-Determining Gene dmy. Animals 2021, 11, 1327. https://doi.org/ 10.3390/ani11051327

Academic Editor:

Mariasilvia D'Andrea

Received: 8 April 2021

Accepted: 3 May 2021

Published: 6 May 2021

Publisher's Note: MDPI stays neutral with regard to jurisdictional claims in published maps and institutional affiliations.

Copyright: (c) 2021 by the authors. Licensee MDPI, Basel, Switzerland. This article is an open access article distributed under the terms and conditions of the Creative Commons Attribution (CC BY) license (https:/ / creativecommons.org/licenses/by/ $4.0 /)$.
Simple Summary: $d m y$ is considered to be the male-determining gene in Japanese medaka (Oryzias latipes) and Hainan medaka (Oryzias. curvinotus), both of which have the XX/XY sex-determination system. Here, we found a group of medaka in the Sanya River (named SY-medaka) and confirmed that SY-medaka belongs to O. curvinotus by morphological characteristics and mitochondrial phylogenetic analysis. Through genetic sex identification, genome re-sequencing and gonadal transcriptome analysis, it was preliminary confirmed that SY-medaka did not contain $d m y$. Our results provide a basis for further studies of the mechanism underlying sex determination in Oryzias and functional genomics and reproduction biology in O. curvinotus.

Abstract: Hainan medaka (Oryzias curvinotus) is distributed in the coastal waters of the South China Sea and is able to adapt to a wide range of salinities. In this study, we characterized O. curvinotus in Sanya River (SY-medaka), which lacks $d m y$ (a male sex-determining gene in O. latipes and $O$. curvinotus). In a comparison of SY-medaka and Gaoqiao medaka (GQ-medaka), the morphological difference between the two populations does not reach the subspecies level and they can be considered two geographic populations of $O$. curvinotus. A mitochondrial cytochrome oxidase subunit I (CoI) sequence alignment showed that the sequence identities between SY-medaka and other geographic populations of $O$. curvinotus are as high as $95 \%$. A phylogenetic analysis of the mitochondrial genome also indicated that SY-medaka belongs to O. curvinotus. Molecular marker-based genetic sex assays and whole genome re-sequencing showed that SY-medaka does not contain dmy. Further, in RNA-Seq analyses of the testis and ovaries of sexually mature SY-medaka, dmy expression was not detected. We speculate that high temperatures resulted in the loss of $d m y$ in SY-medaka during evolution, or the lineage has another sex-determining gene. This study provides a valuable dataset for elucidating the mechanism underlying sex determination in Oryzias genus and advances research on functional genomics or reproduction biology in O. curvinotus.

Keywords: Oryzias curvinotus; dmy; sex determining gene; RNA-Seq

\section{Introduction}

Hainan medaka (Oryzias curvinotus) has recently been reported along the coast of the South Sea [1,2]. O. curvinotus is able to survive in both hyperosmotic and hypoosmotic 
environments and is an emerging model for analyses of osmotic adaptation as well as for marine environmental and toxicological studies [1]. Previous studies have shown that O. curvinotus employs a XX/XY sex chromosome system and has the sex-determining gene dmy [3-5]. Accordingly, it has the potential to become a model for studies of sex determination and differentiation in marine fish.

Various sex-determining genes in fish have been identified, including $d m y$ (Y-specific DM-domain) in Oryzias latipes and O. curvinotus [3-6], sdy (sexually dimorphic on the Y chromosome) in Oncorhynchus mykiss [7], amhr2 (anti-Mullerian hormone receptor type II) in Takifugu rubripes [8], amhy in Odontesthes hatcheri [9], gsdf (gonadal soma-derived growth factor on the Y chromosome) in Oryzias luzonensis [10], and dmrt1 (Doublesex and mab-3 related transcription factor 1) in Cynoglossus semilaevis [11,12]. However, the sex-determining genes in most fish are still unclear. RNA-seq is a rapid method to obtain gene expression data in the absence of sequenced genomes [13]. This approach has been used to identify sex-related genes and for transcriptome profiling in relation to gonadal development and gametogenesis in fish, including Oreochromis niloticus [14], Scatophagus argus [15], and Sillago sihama [16]. We found a population of medaka (named SY-medaka) in the Sanya River, southern Hainan province, which is suspected to be O. curvinotus.

Morphological divergences analysis is the traditional and one of the most basic methods for the study of fish taxonomy [17-19]. Mitochondrial genes are highly conserved and can be used for species classification [20]. Phylogenetic analyses based on mitochondrial sequences can be used to identify genetic relationships among species [21,22]. In this study, we collected SY-medaka and determined whether it belongs to O. curvinotus by morphological and mitochondrial genome analyses. We also evaluated sex-specific molecular markers in SY-medaka and compared the transcriptomes of the testis and the ovaries in the population for the first time.

\section{Materials and Methods}

\subsection{Ethics Statement}

The work described in this article has been carried out in compliance with committee at the Guangdong Ocean University. All samples were obtained under MS222 anesthesia.

\subsection{Collection of Fish and Samples}

Wild sexually mature medaka (body length $29.25 \pm 1.5 \mathrm{~mm}$; body weight $0.27 \pm 0.03 \mathrm{~g}$, salinity, $30 \mathrm{ppt}$ ) were collected from Sanya River (named SY-medaka), Hainan Province, China $\left(18^{\circ} 14^{\prime} 29.50^{\prime \prime} \mathrm{N}, 109^{\circ} 30^{\prime} 29.76^{\prime \prime} \mathrm{E}\right)$. The wild individuals were desalinated in the laboratory, cultured in fresh water for 30 days, and anesthetized by soaking with MS222 $(20 \mu \mathrm{g} / \mathrm{L})$ for anatomic dissection. The physiological sex of SY-medaka was determined by anatomical gonadal type, testis and ovaries were dissected and frozen in liquid nitrogen for RNA extraction, and fins were placed in alcohol for DNA extraction.

\subsection{Morphological Traits and Molecular Species Identification}

Nineteen morphological traits of SY-medaka and GQ-medaka (medaka collected from the National Mangrove Nature Reserve in Gaoqiao, Guangdong Province, China) $\left(21^{\circ} 36^{\prime} 24^{\prime \prime} \mathrm{N}, 109^{\circ} 47^{\prime} 8^{\prime \prime} \mathrm{E}\right)$ [23] were compared and analyzed (Table 1). Covariance analysis was used to compare the morphology of the two populations of medaka. In the analysis of covariance, body length was taken as the covariable, and other measurable traits were corrected and compared, and then unified analysis of variance was conducted. The coefficient difference $(\mathrm{Cd})$ was calculated by the following formula: $\mathrm{Cd}=(\mathrm{M} 1-\mathrm{M} 2) /(\mathrm{S} 1+\mathrm{S} 2)$. $\mathrm{M} 1, \mathrm{M} 2, \mathrm{~S} 1$, and S2 respectively correspond to the mean value and standard deviation (sd) of the trait indexes of the two populations. A total of 50 SY-medaka and 41 GQ-medaka were counted and measured. Measurements were performed following the methods of Zhang et al. [24]. SPSS18.0 (IBM, New York, NY, USA) was used to perform one-way analysis of variance. 
Table 1. Comparison of morphological traits between GQ-medaka and SY-medaka.

\begin{tabular}{cccc}
\hline Countable Traits & GQ-Medaka & SY-Medaka & Variable Coefficient \\
\hline dorsal fin ray counts & $6.02 \pm 0.15$ & $6.04 \pm 0.19$ & 0.05 \\
anal fin ray counts & $19.49 \pm 0.87$ & $19.71 \pm 0.80$ & 0.13 \\
pectoral fin ray counts & $8.09 \pm 0.29$ & $8.12 \pm 0.32$ & 0.05 \\
caudal fin ray counts & $18.51 \pm 0.69 \mathrm{~b}$ & $19.31 \pm 1.02 \mathrm{a}$ & 0.47 \\
ventral fin ray counts & $6.00 \pm 0.00$ & $6.00 \pm 0.00$ & \\
measurable traits & & & \\
Body weight $/ \mathrm{g}$ & $0.203 \pm 0.06$ & $0.204 \pm 0.04$ & 0.01 \\
Total length/mm & $27.71 \pm 2.25 \mathrm{a}$ & $27.59 \pm 1.83 \mathrm{~b}$ & 0.03 \\
Head length/mm & $5.20 \pm 0.62 \mathrm{~b}$ & $5.44 \pm 0.50 \mathrm{a}$ & 0.2 \\
Snount length/mm & $1.21 \pm 0.25$ & $1.29 \pm 0.22$ & 0.17 \\
Eye orbit diameter/mm & $2.06 \pm 0.27$ & $2.00 \pm 0.22$ & 0.12 \\
Maximum depth of body/mm & $5.01 \pm 0.72$ & $5.12 \pm 0.44$ & 0.09 \\
Length of caudal fin $/ \mathrm{mm}$ & $4.32 \pm 0.35$ & $4.21 \pm 0.31$ & 0.17 \\
Tips of snout to anus $/ \mathrm{mm}$ & $12.22 \pm 0.94$ & $12.04 \pm 0.80$ & 0.1 \\
Tip of snout to dorsal fin $/ \mathrm{mm}$ & $13.14 \pm 0.98 \mathrm{a}$ & $12.63 \pm 0.92 \mathrm{~b}$ & 0.27 \\
Caudal peduncle length/mm & $4.19 \pm 0.33$ & $4.17 \pm 0.23$ & 0.04 \\
Caudal peduncle depth/mm & $2.09 \pm 0.25 \mathrm{~b}$ & $2.24 \pm 0.18 \mathrm{a}$ & 0.35 \\
Length of dorsal fin/mm & $3.30 \pm 0.67 \mathrm{~b}$ & $3.68 \pm 0.67 \mathrm{a}$ & 0.28 \\
Length of base of anal fin $/ \mathrm{mm}$ & $2.78 \pm 0.35 \mathrm{~b}$ & $2.96 \pm 0.41 \mathrm{a}$ & 0.23 \\
Length of pectoral fin/mm & $3.90 \pm 0.41 \mathrm{~b}$ & $4.36 \pm 0.37 \mathrm{a}$ & 0.58 \\
\hline
\end{tabular}

Note: The value in the table is mean \pm standard deviation (sd), different letters $(a, b)$ indicate significant differences between GQ-medaka (Gaoqiao medaka) and SY-medaka (medaka in the Sanya River).

Genomic DNA was extracted from each sample using the TIANamp Marine Animals DNA Kit (TIANGEN, Beijing, China) according the instructions. DNAs from 10 females and 10 males of SY-medaka were used to detect the mitochondrial cytochrome oxidase subunit I (COI)sequence for species identification. COI-F/COI-R primers (Table S1) were used for PCR amplification, and the PCR program was as follows: $95^{\circ} \mathrm{C}$ for $5 \mathrm{~min} ; 35$ cycles of $95^{\circ} \mathrm{C}$ for $30 \mathrm{~s}, 50{ }^{\circ} \mathrm{C}$ for $30 \mathrm{~s}$, and $72{ }^{\circ} \mathrm{C}$ for $1 \mathrm{~min}$; then $72{ }^{\circ} \mathrm{C}$ for $7 \mathrm{~min}$ for extension. PCR products were sent to Genewiz Company (Suzhou, China) for sequencing. DNAs from 100 females and 130 males of SY-medaka were used for genetic sex determination, using GQ-medaka as a positive control. One pair of primers, Ocsex-F/Ocsex-R (Table S1), which can amplify different size fragments of $d m r t 1$ and $d m y$ in O. curvinotus, was used to detect the genetic sex of SY-medaka [25]. The reaction conditions were as follows: $95^{\circ} \mathrm{C}$ for $5 \mathrm{~min} ; 35$ cycles of $95^{\circ} \mathrm{C}$ for $30 \mathrm{~s}, 60^{\circ} \mathrm{C}$ for $30 \mathrm{~s}$, and $72{ }^{\circ} \mathrm{C}$ for $1 \mathrm{~min}$; then $72{ }^{\circ} \mathrm{C}$ for $7 \mathrm{~min}$ for extension. PCR products were examined by electrophoresis on $1.5 \%$ agarose gels. Using this method, if a PCR product yielded a single band by electrophoresis, the individual was identified as a genetic female $(X X)$; if a double band was obtained, the individual contained $d m y$ and was a genetic male (XY) [25].

\subsection{Genome Re-Sequencing}

Fifteen SY-medaka and fifteen GQ-medaka individuals were used for genome resequencing. The genomic DNA was extracted with a total amount of $1.5 \mu \mathrm{g}$ per sample for library construction. Sequencing libraries were generated using Truseq Nano DNA HT Sample preparation Kit (Illumina, California, CA, USA) following manufacturer's recommendations, and index codes were added to attribute sequences to each sample. Briefly, the DNA sample was fragmented by sonication to a size of $350 \mathrm{bp}$, then DNA fragments were end polished, A-tailed, and ligated with the full-length adapter for Illumina sequencing with further PCR amplification. At last, PCR products were purified and libraries were analyzed for size distribution by Agilent2100 Bioanalyzer and quantified using real-time PCR. Whole genomes of 30 samples were sequenced based on the Illumina Hiseq PE150 platform. The Hiseq sequencing was converted to raw data by Base Calling, and the lower-quality reads (Reads with $\geq 10 \%$ unidentified nucleotides $(\mathrm{N}) ;>10 \mathrm{nt}$ aligned 
to the adaptor, allowing $\leq 10 \%$ mismatches; $>50 \%$ bases having phred quality $<5$; and putative PCR duplicates generated in the library construction process) were removed to obtain clean data. Clean data was compared to the reference genome by BWA software (parameter: mem-t 4-k 32-M) [26], and duplicates were removed by SamTools (parameter: rMDUP) [27].

\subsection{RNA Isolation, cDNA Library Preparation, and Sequencing}

To further determine whether SY-medaka expresses $d m y$, RNA-Seq was performed using the testes and ovaries of SY-medaka. The gonadal development of SY-medaka was analyzed by a histological analysis of sections. For males, testes from four individuals were pooled to obtain one sample. For females, the ovaries of one individual were used as one sample. One sample was used to construct one cDNA library, and eventually three testis libraries and three ovary libraries were obtained. Total RNA isolation and cDNA library preparation were completed by BGI (Beijing, China). For the details of the procedure, refer to Lin et al. [28]. The library was sequenced using the BGISEQ-500 sequencing platform (MGI Tech Co., Ltd., Shenzhen, China) and paired-end 100-bp reads were generated. All raw data have been submitted to the CNGB Nucleotide Sequence Archive (CNSA) under the accession number CNP0000963.

\subsection{De Novo Assembly and Functional Annotation}

Clean data were obtained by removing low-quality reads (i.e., reads with more than $20 \%$ of the bases having a Phred score lower than 15), reads with adaptors, and reads with unknown bases ( $\mathrm{N}$ bases more than $5 \%$ ). Those clean reads were de novo assembled using Trinity (v2.0.6), and gene family clustering was performed using Tgicl (v2.0.6) to obtain the final unigenes for functional annotation. BLASTn (v2.2.23, http:/ /blast.ncbi. nlm.nih.gov/Blast.cgi (accessed on 8 October 2018)) with an E-value threshold of $1 \times 10^{-5}$ was used to query the unigenes against Nt (NCBI non-redundant nucleotide sequences). Diamond (v0.8.31, https:/ / github.com/bbuchfink/diamond (accessed on 14 August 2018)) with an E-value cut-off of $1 \times 10^{-5}$ was used to query the unigenes against the NR (NCBI non-redundant protein databases), KOG (eukaryotic orthologous group database), KEGG (Kyoto Encyclopedia of Genes database), and Swiss-Prot protein databases. Gene ontology (GO) annotation of unigenes was performed using Blast2GO based on the National Center for Biotechnology Information (NCBI) NR database annotation results [29].

\subsection{Differential Gene Expression Analysis and Quantitative Real-Time PCR ( $P P C R$ ) Validation}

Clean reads were mapped to unigenes using Bowtie2 [30], and the gene expression level was calculated using RNA-Seq by Expectation Maximization (RSEM) [31]. The fragments per kilobase per million reads (FPKM) method was used to quantify gene expression levels. The differentially expressed genes (DEGs) were detected using NOIseq as described by Tarazona et al. [32]. Unigenes with Fold Change $\geq 2.00$ and Probability $\geq 0.9$ between the testes and ovaries were considered DEGs. Twenty genes were selected to verify the accuracy of the sequence assembly by PCR and sequencing, and 20 were used for the validation of the transcript expression profile by quantitative real-time PCR (qPCR). qPCR primers (Table S1) were designed according to transcriptomic results, and PCR amplification and clone sequencing were performed with each pair of qPCR primers. qPCR was performed using the Roche LightCycler 96 system (Roche, Forrentrasse, Switzerland). qPCR amplification conditions were as follows: $180 \mathrm{~s}$ at $95^{\circ} \mathrm{C}$ for pre-incubation, followed by 40 cycles at $95^{\circ} \mathrm{C}(15 \mathrm{~s}), 60^{\circ} \mathrm{C}(15 \mathrm{~s})$, and $72{ }^{\circ} \mathrm{C}(30 \mathrm{~s})$. The combination of eef1b and rps4x was used as the internal reference, and three technical repetitions were used for each sample [23]. The transcript abundance of each gene relative to the corresponding solvent control was $\log _{2}$-transformed according to the ${ }^{2-\Delta \Delta C t}$ method [33]. 


\section{Results}

\subsection{Morphological and Molecular Analyses of SY-Medaka}

We compared countable and measurable traits of SY-medaka and GQ-medaka by one-way analysis of variance. Except for the significant difference in caudal fin ray counts between the SY-medaka (19.31 \pm 1.02$)$ and the GQ-medaka $(18.51 \pm 0.69)$, there were no differences in the other four traits between the two groups (Figure S1, Table 1). For measurable traits, total length, head length, tip of the snout to dorsal fin, caudal peduncle depth, length of the dorsal fin, length of the base of the anal fin, and length of the pectoral fin differed significantly between groups $(p<0.05)$. The coefficient of variation between the two groups was highest for the length of the pectoral fin at 0.58 (Table 1).

COI of SY-medaka was cloned and sequenced (Table S2). The sequence identity between SY-medaka and GQ-medaka was as high as 95\% (data not shown). Furthermore, a 16,667-bp mitochondrial genome was assembled from RNA-seq data, including 13 proteincoding genes and 22 tRNA genes (Figure 1A). Sequence alignment by BLAST (https: / /blast.ncbi.nlm.nih.gov/Blast.cgi) showed that SY-medaka has maximum identities with O. curvinotus (99.33\%), followed by O. luzonensis (88.25\%) and O. latipes (83.89\%). An ML phylogenetic tree was constructed based on the complete mitochondrial genome sequence of 11 medaka fishes, with zebrafish as the outgroup. The results showed that SY-medaka and O. curvinotus clustered together, and O. luzonensis, O. latipes, and O. sinensis formed a separate cluster (Figure 1B). Furthermore, the evolutionary divergence analysis of mitochondrial sequences among the above-mentioned fish indicated that the genetic distance between SY-medaka and O. curvinotus was only 0.005, while the genetic distance between SY-medaka and O. luzonensis was 0.095, which was similar to the genetic distance between O. curvinotus and O. luzonensis (0.094) (Table S3).

A

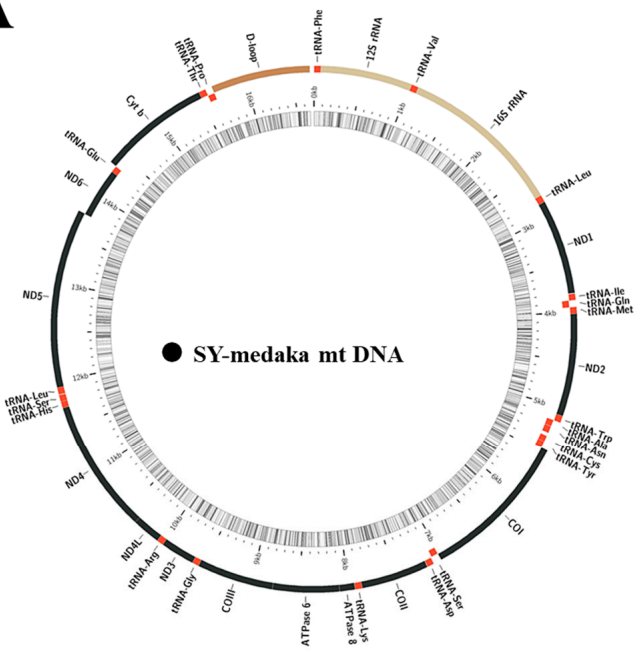

B
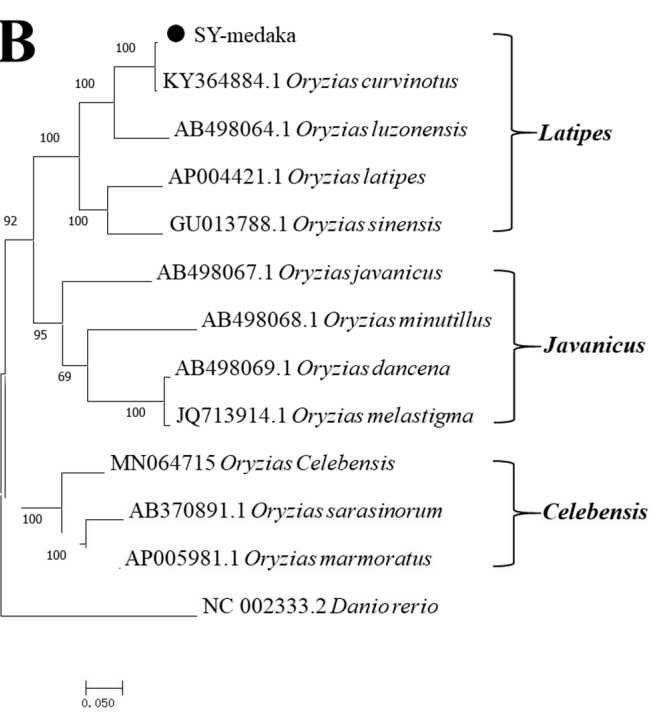

Figure 1. Structure of the mitochondrial genome of SY-medaka (medaka in the Sanya River) (A) and phylogenetic tree analysis of the mitochondrial sequence of Oryzias (B). The phylogenetic tree was constructed using MEGA 6.0 with the maximum likelihood method. Black point indicates SY-medaka.

\subsection{Genetic Sex Identification of SY-Medaka}

Genetic assays were performed with the sex-specific primers Ocsex-F and -R [25]. After amplification, only one band was detected for both male and female SY-medaka. However, in GQ-medaka, females had a single band and males had two bands (Figure 2A). The dmy locus was not detected in any of the tested individuals of SY-medaka. Further, we analyzed re-sequencing data for 15 SY-medaka and 15 GQ-medaka. The males in the two groups showed differentiation in $d m y$. The coverage of $d m y$ per male and female individual in SY-medaka was consistent and similar to that of female GQ-medaka (Figure 2B). Dmrt1 
was selected as a control for $d m y$ coverage analysis to verify the re-sequencing data was reliable, the coverage rate of $d m r t 1$ in SY-medaka and GQ-medaka was almost the same, and there was no significant difference between females and males (Figure S2).
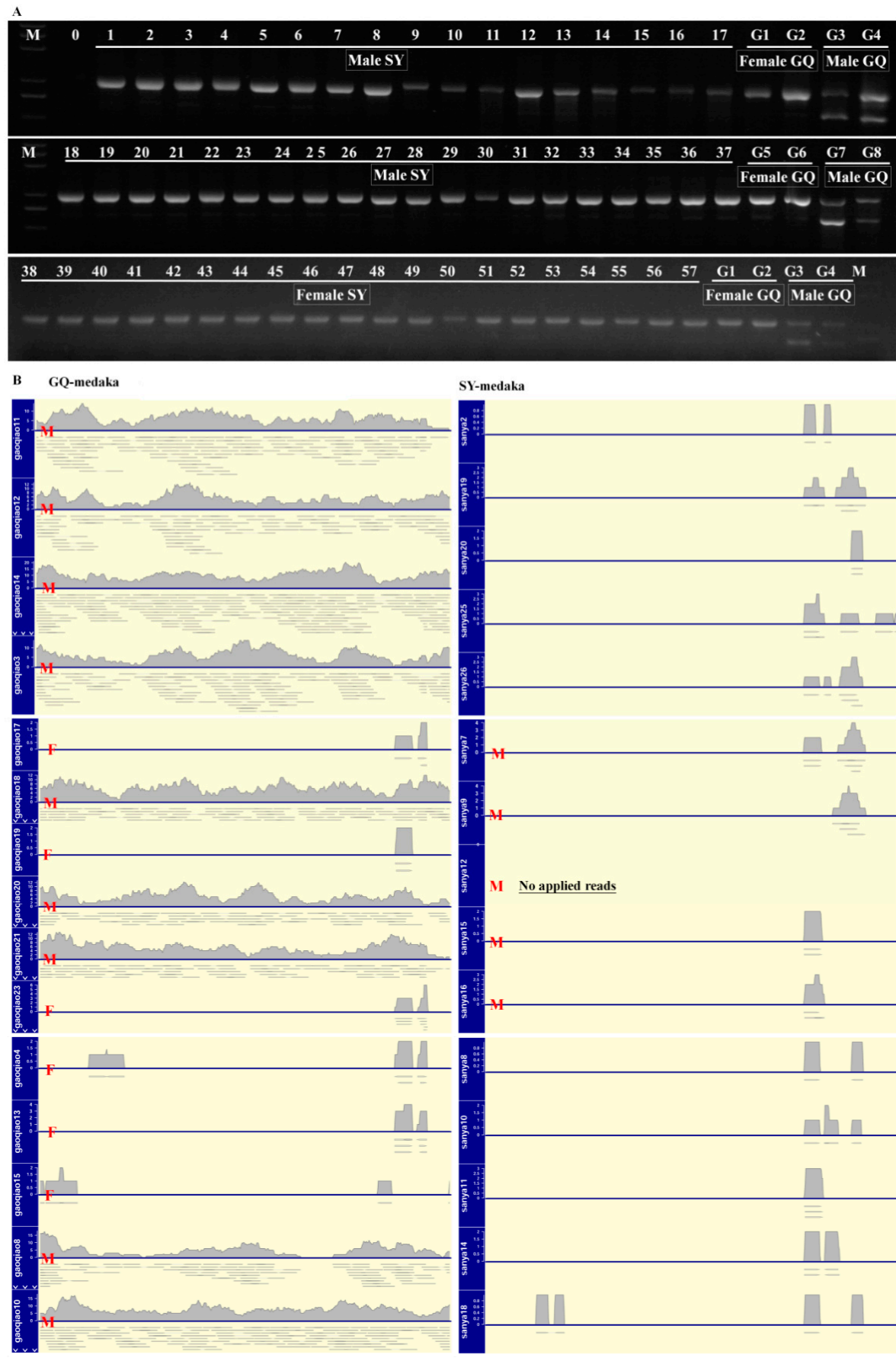

Figure 2. Genetic sex identification of O.curvinotus. (A) Gel electrophoresis results of PCR products amplified from the genome of O. curvinotus by primer Ocsex-F/Ocsex-R. Male SY-medaka (medaka in the Sanya River): 1-17 and 18-37. Male GQ-medaka (Gaoqiao medaka): G3, G4, G7, and G8. Female SY-medaka: 38-57. Female GQ-medaka: G1, G2, G5, and G6. 0 is a negative control; M is a DL 5000 DNA marker. (B) Coverage of $d m y$ by whole genome re-sequencing reads of GQ-medaka and SY-medaka. M indicates male and F indicates female. Only five males of SY-medaka $(7,9,12$, 15 , and 16) had complete sexual identification records, unfortunately the sampling records of the remaining 10 samples (six females and four males) were missing, making it impossible to pinpoint the sex of each sample. 


\subsection{RNA-Seq Analysis, Unigene Annotation, and dmy Detection}

The results of gonad histological section showed that the SY-medaka used in this study were sexually mature (Figure S3). A total of $36.69 \mathrm{~Gb}$ of data was generated using the BGISEQ-500 platform (Table S4). Using Trinity, 84,484 unigenes were assembled with an N50 length of $2384 \mathrm{bp}$, and $93.87 \%$ of unigenes $(79,301)$ were annotated (Table S5). By searching against the NR database, 46,375 unigenes matched to known sequences from 430 species, and the top three species were O. latipes $(35,653 ; 76.88 \%)$, Larimichthys crocea (9523; 15.46\%), and Lates calcarifer (3210; 5.21\%) (Figure S4). By searching the annotation results, dmy mRNA expression was not detected. The nucleic acid and amino acid sequences of O. curvinotus dmy (GenBank accession nos: BAC65995.1) were used to generate a local BLAST alignment of transcriptome data, and dmy was not included in the hits.

\subsection{Differentially Expressed Gene Identification and qPCR Validation}

In this study, 33,192 unigenes were identified as differentially expressed genes (DEGs) between the testes and ovaries (Figure 3A). Among these, 15,710 unigenes were upregulated in the testis and 17,482 unigenes were up-regulated in the ovaries. Many of these DEGs were involved in sex determination and gonadal development, such as doublesex and mab-3 related transcription factor 1 (dmrt1), forkhead box protein L2 (foxl2), cytochrome P450s (cyps), izumo sperm-egg fusion protein 1 (izumo1), sperm flagellar proteins (spefs), wingless-type mice mammary tumor virus (MMTV) integration site family (wnts), zona pellucida sperm-binding proteins (zps), SRY (sex determining region Y)-box (soxs), and anti-Müllerian hormone (amh) (Table S6). The sequencing results for 22 PCR products (20 target genes and 2 references genes) indicated that the assembled unigenes were correct (data not shown). Further, we validated the gene expression patterns obtained by RNA-Seq using qPCR. The qPCR results were consistent with those of the RNA-Seq analysis (Figure 3B,C).
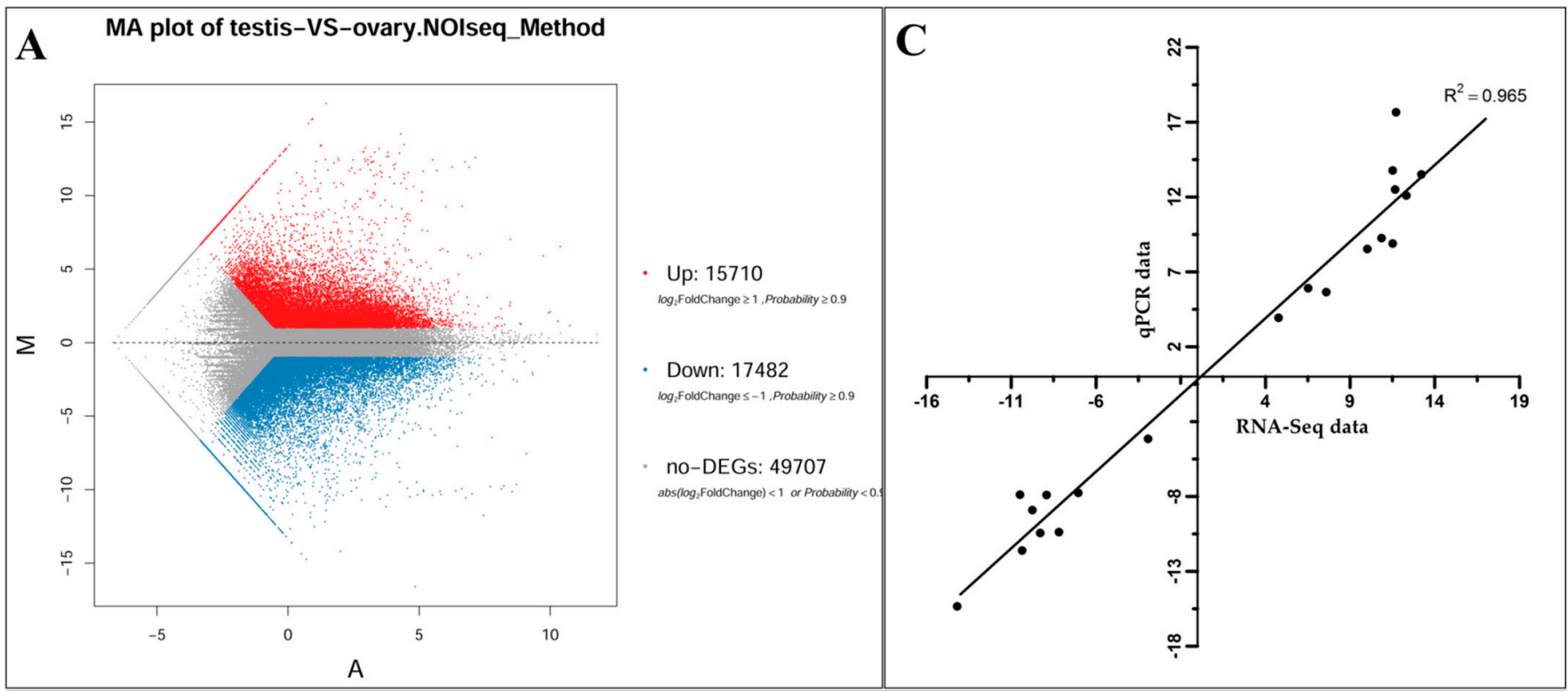

Figure 3. Cont. 


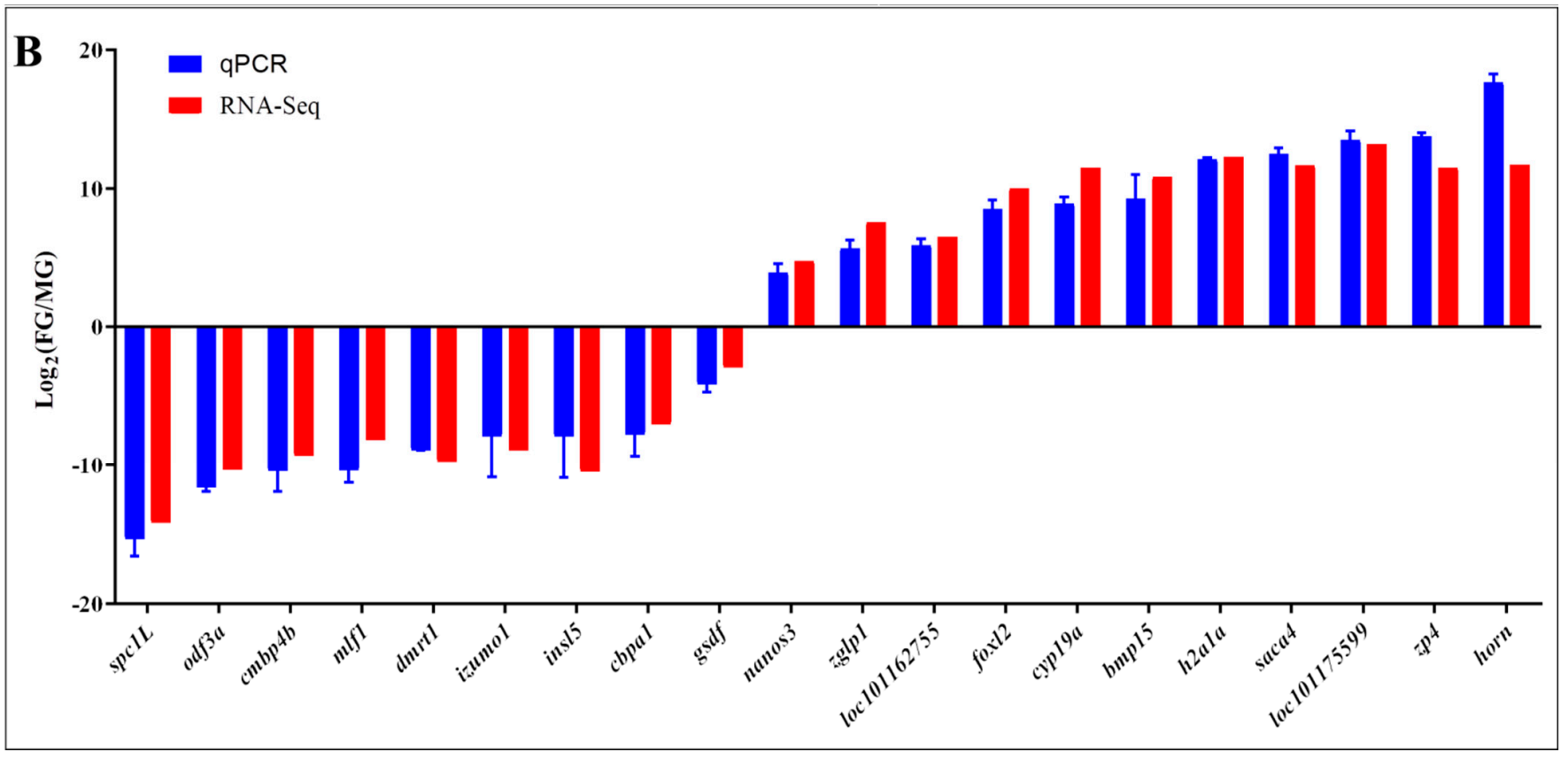

Figure 3. (A) MA plot of differentially expressed genes between the testis and ovary. (B) Validation of RNA-Seq data using qPCR. The RNA-Seq results are displayed as $\log _{2}$ Fold Change (ovary/testis), ( $n=3$ for each sex). qPCR results were evaluated by relative expression using eef1b and $r p s 4 x$ as reference genes and the optimized comparative Ct $\left(2^{-\Delta \Delta C t}\right)$. (C) Consistency of $\log _{2}$ Fold Change(ovary/testis) between RNA-Seq data ( $x$-axis) and qPCR data ( $y$-axis) was high $\left(R^{2}=0.965\right)$ based on 20 genes.

\section{Discussion}

\subsection{SY-Medaka Belongs to O. curvinotus.}

The morphological traits of fish are often used for species identification. The value 1.28 is a critical correlation coefficient for subspecies classification [34]. When the coefficient of variation of morphological traits between two populations exceeds this threshold, the populations can be considered subspecies; this measure only considers the morphological difference between geographical groups. SY-medaka and GQ-medaka have similar appearance (Figure S1). The variation of number of caudal fins was 0.47 , the correlation coefficient of the length of pectoral fin was 0.58 (Table 1), both of them were less than the value of 1.28. Therefore, in terms of morphological characteristics, the differentiation between SY-medaka and GQ-medaka has not reached the subspecies level, and they can be considered different geographical groups of the same species.

Mitochondrial phylogenetic analyses of medaka indicate that the species can be divided into three major clades, referred to as the latipes, javanicus, and celebensis species groups (Figure 1B) [35,36]. Hellberg et al. (2009) proposed that when using mitochondrial genes as barcodes for the identification of animal species, the intraspecific genetic distance should not be more than 0.02 , and the interspecific genetic distance should be significantly greater than the intraspecific distance [37]. In this study, the genetic distance between SY-medaka and O. curvinotus was only 0.005 , much lower than the genetic distance between SY-medaka and O. luzeonensis of 0.095. Combined with morphological results and mitochondrial analysis, we determined that the SY-medaka population belongs to O. curvinotus.

\subsection{SY-Medaka Does Not Contain dmy}

The first sex-determining gene ( $d m y)$ was discovered in O. latipes [3], making it an ideal model for studies of sex determination and differentiation. Researchers have identified dozens of species in Oryzias, such as O. latipes, O. sinensis, O. curvinotus, O. luzonensis, O. dancena, O. celebensis, O. mekongensis, O. javanicus, and O. melastigma [38]. Previous studies have indicated that the sex chromosome type of O. curvinotus is $\mathrm{XX} / \mathrm{XY}$, and 
$d m y$ is considered the sex-determining gene in this species [38]. However, SY-medaka may be an exception. In this study, nearly 300 individuals of SY-medaka were used for detection of $d m y$, and the results were negative (Figure 2A). Whole genome re-sequencing is currently the most comprehensive technology for determining the genetic basis of important traits [39]. In this study, whole genome re-sequencing reads for males and females of SY-medaka show low coverage of $d m y$, consistent with the re-sequencing results for female GQ-medaka (Figure 2B). In summary, based on molecular markers and whole genome re-sequencing, SY-medaka does not contain $d m y$. Previous studies have detected transcripts of $d m y$ in the testis of mature O. latipes and O. curvinotus $[3,5,6]$. In this study, the nucleotide and protein sequences of O. curvinotus dmy were used for local BLAST searches, and $d m y$ was not found, indicating that $d m y$ expression is absent in SY-medaka.

\subsection{Genes Related to Sex Determination and Gonadal Development in SY-Medaka}

RNA-Seq analyses of gonads can be useful for the identification sex-related genes and candidate sex determination genes. We performed the first RNA-Seq analysis of SY-medaka gonads. The annotation rate ( $93.87 \%$ of 84,484 unigenes) was higher than those of the gonad transcriptomes of S. sihama (46.06\% of 74,038 unigenes) [16] and S. argus (61.51\% of 136,561$)$. In a search against the NR database, $76.88 \%$ of annotated unigenes were found to be homologous to genes in O. latipes (Figure S4), reflecting the close evolutionary relationship between O. curvinotus and O. latipes [38]. Numerous DEGs related to male sex determination or testis development were identified by RNA-Seq, including $d m r t 1$, gsdf, sox9, amh, ars, smcs, hsd17b, hdacs, and izumo1 (Table S6) [3,4,11,12,40-44]. In addition, several genes involved in female sex determination or ovarian development also were identified, such as foxl2, cyp19a1a, cyps, zps, gdf9, zar 1, bmps, and zglp $1[15,45-50]$. These findings provide a basis for research on sex determination, differentiation, and reproduction in O. curvinotus.

\subsection{Lack of dmy in the SY-Medaka Genome}

Fish sex determination is affected by genetic and environmental factors, and an important environmental factor is temperature [51]. Fish are sensitive to environmental conditions during gonad differentiation, and abnormal water temperatures can determine the direction of gonad differentiation $[52,53]$. High temperature often leads to the masculinization of fish [54-57]. Sanya is located in the tropics with high water temperatures, so we speculate that the SY-medaka lost $d m y$ during evolution (Figure 4). Briefly, XX embryos of SY-medaka developed into males (XX-male) in high-temperature conditions; the $X X$-male, competitive with $X Y$-male, mated with $X X$-female, which can only produce $X X$ type offspring, increasing the ratio of $X X$ in offspring. The ratio of $X X$ in the population of SY-medaka accumulated over generations, which made the value of XX:XY became infinite, explaining the lack of $X Y$ individuals detected in our survey (Figure 4). Additional field surveys and reproduction experiments are needed to verify this hypothesis.

An alternative hypothesis is that SY-medaka has a sex determination gene other than $d m y$. Sex determination mechanisms vary among bony fishes, and species in the genus Oryzias harbor diverse sex-determining genes [38,51]. Three master sex-determining genes have been identified in Oryzias to date. In addition to $d m y$, which acts as a male sexdetermining gene in O. latipes [3,4], gsdfy is a sex-determining gene in O. luzonensis [10], and sox 3 serves as a sex-determining gene in O. dancena, O. marmoratus, and O. profundicola $[30,57,58]$. This diversity has inspired ongoing research on the molecular mechanisms underlying the rewiring of gene regulatory networks that are required to establish new master sex-determining genes $[38,59,60]$. 


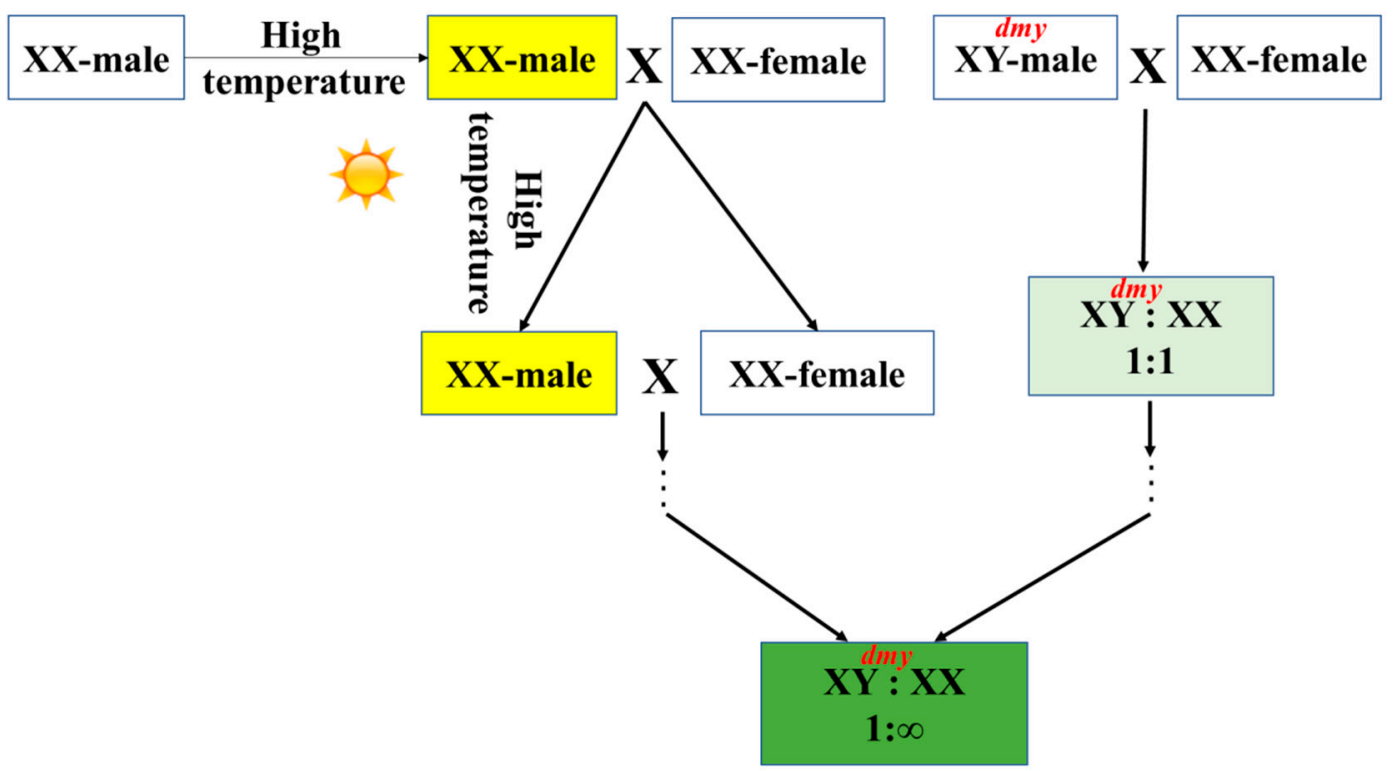

Figure 4. Schematic diagram of the evolution of $d m y$ in SY-medaka (medaka in the Sanya River).

\section{Conclusions}

In conclusion, we identified the SY-medaka population as O curvinotus based on morphological characteristics and a mitochondrial sequence analysis. Interestingly, sexspecific molecular markers and whole genome re-sequencing indicated that the SY-medaka genome lacks $d m y$. We also completed the first RNA-Seq analysis of the testis and ovary of SY-medaka and identified numerous DEGs related to sex determination and gonadal development. The RNA-Seq results also support the lack of $d m y$ mRNA expression in SY-medaka. We speculate that the absence of $d m y$ in SY-medaka can be explained by the high-temperature conditions, resulting in gene loss during evolution, or by the existence of another sex-determining gene. These findings provide a basis for further studies of the mechanism underlying sex determination in Oryzias and for studies focused on functional genomics and reproduction biology in O. curvinotus.

Supplementary Materials: The following are available online at https://www.mdpi.com/article/ 10.3390/ani11051327/s1, Figure S1: O. curvinotus form Gaoqiao and Sanya; Figure S2: Coverage of $d m r t 1$ by whole genome re-sequencing reads of GQ-medaka and SY-medaka; Figure S3: Histological section of testis and ovary of SY-medaka; Figure S4: Distribution of homologous species of SY-medaka unigenes, Table S1: The sequences of primers used in this study; Table S2: The -sequencing results of 15 SY-medaka; Table S3: Estimates of evolutionary divergence between sequences; Table S4: Summary statistics of testes and ovaries transcriptome data of O. curvinotus; Table S5: O. curvinotus transcriptome reference assembly and annotation statistics; Table S6: The expression patterns of some DEGs related to gonad development and reproduction.

Author Contributions: Conceptualization, Z.D., C.S., and Z.W.; Data curation, Z.D., X.L., and Z.Y.; Methodology, Z.D., X.L. and C.W.; Software Z.D., X.L., and Q.W.; Supervision, C.S. and Y.G. and Z.W.; Writing - original draft and Writing - editing, Z.D., Q.W., C.S. and Z.W.; Funding acquisition, Z.D. and Y.G. All authors have read and agreed to the published version of the manuscript.

Funding: This work was funded by the National Natural Science Foundation of China (41806195, 31972794), Special innovation projects of colleges and universities in Guangdong province (2018KTSCX087, 230419055), Nanhai Scholar Project of GDOU (QNXZ201807, 201903), Guangdong South China Sea Key Laboratory of Aquaculture for Aquatic Economic Animals, Guangdong Ocean University (KFKT2019ZD03) and State Laboratory of Developmental Biology of Freshwater Fish (2020KF004).

Institutional Review Board Statement: The study was approved by Animal Research and Ethics Committee of Guangdong Ocean University (201903003). 
Data Availability Statement: All data generated or analyzed in this study are included in the submitted article and its supplementary information files. The raw RNA-Seq data were deposited at the CNGB Nucleotide Sequence Archive (CNSA) under the accession number CNP0000963 (https://db.cngb.org/search/?q=CNP0000963+).

Acknowledgments: The authors thank Jin Gao for helping with data analyses of this manuscript.

Conflicts of Interest: The authors declare no conflict of interest. The funders had no role in the design of the study; in the collection, analyses, or interpretation of data; in the writing of the manuscript, or in the decision to publish the results.

\section{References}

1. Hayakawa, H.; Le, Q.D.; Kinoshita, M.; Takehana, Y.; Sakuma, K.; Takeshima, H.; Kojima, S.; Naruse, K.; Inoue, K. Genetic similarity of the Hainan medaka populations collected from hyper- and hypo-osmotic environments in northern Vietnam. Ocean. Sci. J. 2015, 50, 231-235. [CrossRef]

2. Wang, Z.D.; Long, S.S.; Liao, J.; Huang, C.Q.; Zhang, H.R.; Huang, S.K.; Zhang, Y.P.; Liu, L.; Guo, Y.S. Complete mitogenome of Hainan medaka Oryzias curvinotus (Teleostei: Beloniformes and transcriptional differences between male and female liver. Mitochondrial DNA B 2017, 2, 157-158. [CrossRef]

3. Matsuda, M.; Nagahama, Y.; Shinomiya, A.; Sato, T.; Matsuda, C.; Kobayashi, T.; Morrey, C.E.; Shibata, N.; Asakawa, S.; Shimizu, N.; et al. DMY is a Y-specific DM-domain gene required for male development in the medaka fish. Nature. 2002, 417, 559-563. [CrossRef]

4. Matsuda, M.; Nagahama, Y.; Kobayashi, T.; Matsuda, C.; Hamaguchi, S.; Sakaizumi, M. The sex determining gene of medaka: A Y-specific DM domain gene (DMY is required for male development. Fish Physiol. Biochem. 2003, 28, 135-139. [CrossRef]

5. Matsuda, M.; Sato, T.; Toyazaki, Y.; Nagahama, Y.; Hamaguchi, S.; Sakaizumi, M. Oryzias curvinotus has DMY, a gene that is required for male development in the medaka, O. latipes. Zool. Sci. 2003, 20, 159-161. [CrossRef]

6. Kato, M.; Takehana, Y.; Sakaizumi, M.; Hamaguchi, S. A sex-determining region on the Y chromosome controls the sex-reversal ratio in interspecific hybrids between Oryzias curvinotus females and Oryzias latipes males. Heredity 2010, 104, 191-195. [CrossRef] [PubMed]

7. Yano, A.; Guyomard, R.; Nicol, B.; Jouanno, E.; Quillet, E.; Klopp, C.; Bouchez, O.; Fostier, A.; Guiguen, Y. An immune-related gene evolved into the master sex-determining gene in rainbow trout, Oncorhynchus mykiss. Curr. Biol. 2012, 22, 1423-1428. [CrossRef]

8. Kamiya, T.; Kai, W.; Tasumi, S.; Oka, A.; Matsunaga, T.; Mizuno, N.; Fujita, M.; Suetake, H.; Suzuki, S.; Hosoya, S.; et al. A trans-species missense SNP in amhr2 is associated with sex determination in the tiger pufferfish, Takifugu rubripes (Fugu). PLoS Genet. 2012, 8, e1002798. [CrossRef]

9. Hattori, R.S.; Strüssmann, C.A. A Y-linked anti-Müllerian hormone duplication takes over a critical role in sex determination. $P$. Natl. Acad. Sci. 2012, 109, 2955. [CrossRef] [PubMed]

10. Myosho, T.; Otake, H.; Masuyama, H.; Matsuda, M.; Kuroki, Y.; Fujiyama, A.; Naruse, K.; Hamaguchi, S.; Sakaizumi, M. Tracing the emergence of a novel sex-determining gene in medaka, Oryzias luzonensis. Genetics 2012, 191, 163-170. [CrossRef] [PubMed]

11. Cui, Z.K.; Liu, Y.; Wang, W.W.; Wang, Q.; Zhang, N.; Lin, F.; Wang, N.; Shao, C.W.; Dong, Z.D.; Li, Y.Z.; et al. Genome editing reveals $d m r t 1$ as an essential male sex-determining gene in Chinese tongue sole (Cynoglossus semilaevis). Sci. Rep. 2017, 7, 42213. [CrossRef]

12. Chen, S.L.; Zhang, G.J.; Shao, C.W.; Huang, Q.F.; Liu, G.; Zhang, P.; Song, W.T.; An, N.; Chalopin, D.; Volff, J.N.; et al. Wholegenome sequence of a flatfish provides insights into ZW sex chromosome evolution and adaptation to a benthic lifestyle. Nat. Genet. 2014, 46, 253-260. [CrossRef]

13. Mortazavi, A.; Williams, B.A.; McCue, K.; Schaeffer, L.; Wold, B. Mapping and quantifying mammalian transcriptomes by RNA-Seq. Nat. Methods 2008, 5, 621-628. [CrossRef]

14. Tao, W.J.; Chen, J.L.; Tan, D.J.; Yang, J.; Sun, L.N.; Wei, J.; Conte, M.A.; Kocher, T.D.; Wang, D.S. Transcriptome display during tilapia sex determination and differentiation as revealed by RNA-Seq analysis. BMC Genom. 2018, 19, 363. [CrossRef] [PubMed]

15. He, F.X.; Jiang, D.N.; Huang, Y.Q.; Mustapha, U.F.; Yang, W.; Cui, X.F.; Tian, C.X.; Chen, H.P.; Shi, H.J.; Deng, S.P.; et al Comparative transcriptome analysis of male and female gonads reveals sex-biased genes in spotted scat (Scatophagus argus). Fish Physiol. Biochem. 2019, 45, 1963-1980. [CrossRef]

16. Tian, C.X.; Liu, Z.Y.; Dong, Z.D.; Huang, Y.; Du, T.; Chen, H.P.; Jiang, D.N.; Deng, S.P.; Zhang, Y.L.; Wanida, S.; et al. Transcriptome analysis of male and female mature gonads of silver sillago (Sillago sihama). Genes 2019, 10, 129. [CrossRef]

17. Cadrin, S.X. Advances in morphometric identification of fishery stocks. Rev. Fish Biol. Fisher. 2000, 10, 91-112. [CrossRef]

18. Frederich, B.; Liu, S.Y.V.; Dai, C.F. Morphological and genetic divergences in a coral reef damselfish, Pomacentrus coelestis. Evol biol. 2012, 39, 359-370. [CrossRef]

19. Liu, X.H.; Song, N.; Liu, H.Y.; Li, Q.H.; Chen, Z.Y.; Yin, L.N.; Wang, Y.P.; Gao, T.X. Preliminary analysis on morphological characteristics of 5 Collichthys lucidus geographical populations. Trans. Oceanol. Limnol. 2015, 2, 59-67. [CrossRef] 
20. Wang, W.; Ma, C.Y.; Chen, W.; Jin, Z.W.; Zhao, M.; Zhang, F.Y.; Liu, Z.Q.; Ma, L.B. Population genetic diversity of mud crab (Scylla paramamosain from southeast coastal regions of China based on mitochondrial COI gene sequence. Gene 2020, $751,144763$. [CrossRef] [PubMed]

21. Tan, M.P.; Jamsari, A.F.J.; Muchlisin, Z.A.; Azizah, M.N.S. Mitochondrial genetic variation and population structure of the striped snakehead, Channa striata in Malaysia and Sumatra, Indonesia. Biochem. Syst. Ecol. 2015, 60, 99-105. [CrossRef]

22. Zhu, S.R.; Fu, J.J.; Wang, Q.; Li, J.L. Identification of Channa species using the partial cytochrome c oxidase subunit I (COI gene as a DNA barcoding marker. Biochem. Syst. Ecol. 2013, 51, 117-122. [CrossRef]

23. Zhang, F.F.; Jiang, H.C.; Jin, J.J.; Qiu, Y.P.; Chen, G.Z. Characteristic re-description of ricefish Oryzias curvinotus from Guangdong, China. Sichuan J. Zool. 2017, 36, 564-571. [CrossRef]

24. Dong, Z.D.; Long, S.S.; Huang, C.Q.; Huang, S.K.; Zhang, N.; Ying, Z.Y.; Guo, Y.S.; Wang, Z.D. A method for rapid identification of genetic sex of Oryzias curvinotus. J. Guangdong Ocean Univ. 2018, 38, 25-29. [CrossRef]

25. Lin, Y.S.; Wang, B.; Wang, N.H.; Ouyang, G.; Cao, H. Transcriptome analysis of rare minnow (Gobiocypris rarus infected by the grass carp reovirus. Fish Shellfish Immunol. 2019, 89, 337-344. [CrossRef]

26. Li, H.; Durbin, R. Fast and accurate short read alignment with Burrows-Wheeler transform. Bioinformatics 2009, 25, 1754-1760. [CrossRef] [PubMed]

27. Li, H.; Handsaker, B.; Wysoker, A.; Fennell, T.; Ruan, J.; Homer, N.; Marth, G.; Abecasis, G.; Durbin, R. The Sequence Alignment/Map format and SAMtools. Bioinformatics 2009, 25, 2078-2079. [CrossRef]

28. Conesa, A.; Gotz, S.; Garcia-Gomez, J.M.; Terol, J.; Talon, M.; Robles, M. Blast2GO: A universal tool for annotation, visualization and analysis in functional genomics research. Bioinformatics 2005, 21, 3674-3676. [CrossRef]

29. Langmead, B.; Salzberg, S.L. Fast gapped-read alignment with Bowtie 2. Nat. Methods 2012, 9, 357-359. [CrossRef] [PubMed]

30. Li, B.; Dewey, C.N. RSEM: Accurate transcript quantification from RNA-Seq data with or without a reference genome. $B M C$ Bioinform. 2011, 12, 16. [CrossRef]

31. Tarazona, S.; Garcia-Alcalde, F.; Dopazo, J.; Ferrer, A.; Conesa, A. Differential expression in RNA-seq: A matter of depth. Genome Res. 2011, 21, 2213-2223. [CrossRef]

32. Dong, Z.D.; Chen, P.S.; Zhang, N.; Huang, S.K.; Zhang, H.R.; Wang, S.R.; Li, X.Y.; Guo, Y.S.; Wang, Z.D. Evaluation of reference genes for quantitative real-time PCR analysis of gene expression in Hainan medaka (Oryzias curvinotus). Gene Rep. 2019, 14, 94-99. [CrossRef]

33. Livak, K.J.; Schmittgen, T.D. Analysis of relative gene expression data using real-time quantitative PCR and the 2(-Delta Delta C (T)). Method 2001, 25, 402-408. [CrossRef]

34. Mayr, E.; Linsley, E.G.; Usinger, R. Methods and Principles of Systematic Zoology; McGraw Hill: New York, NY, USA, 1953; pp. 22-39.

35. Takehana, Y.; Naruse, K.; Sakaizumi, M. Molecular phylogeny of the medaka fishes genus Oryzias (Beloniformes: Adrianichthyidae) based on nuclear and mitochondrial DNA sequences. Mol. Phylogenet Evol. 2005, 36, 417-428. [CrossRef] [PubMed]

36. Mokodongan, D.F.; Yamahira, K. Origin and intra-island diversification of Sulawesi endemic Adrianichthyidae. Mol. Phylogenet Evol. 2015, 93, 150-160. [CrossRef]

37. Hellberg, R.S.; Kawalek, M.D.; Van, K.T.; Shen, Y.L.; Williams-Hill, D.M. Comparison of DNA Extraction and PCR Setup Methods for Use in High-Throughput DNA Barcoding of Fish Species. Food Anal. Methods 2014, 7, 1950-1959. [CrossRef]

38. Leon, H.; Julia, S. The natural history of model organisms: The untapped potential of medaka and its wild relatives. eLife 2019, 8 , e46994. [CrossRef]

39. Liu, H.R.; Zhang, H.; Pan, X.L.; Xu, M.; Huang, J.; He, M.X. A high density genetic map by whole-genome resequencing for QTL fine-mapping and dissecting candidate genes for growth or sex traits in the pearl oyster (Pinctada fucata martensii). Aquaculture 2020, 519, 734839. [CrossRef]

40. Lin, Q.H.; Mei, J.; Li, Z.; Zhang, X.M.; Zhou, L.; Gui, J.F. Distinct and cooperative roles of $a m h$ and $d m r t 1$ in self-renewal and differentiation of male germ cells in zebrafish. Genetics. 2017, 207, 1007-1022. [CrossRef]

41. Nakamura, S.; Watakabe, I.; Nishimura, T.; Picard, J.Y.; Toyoda, A.; Taniguchi, Y.; di Clemente, N.; Tanaka, M. Hyperproliferation of mitotically active germ cells due to defective anti-Mullerian hormone signaling mediates sex reversal in medaka. Development 2012, 139, 2283-2287. [CrossRef] [PubMed]

42. Jiang, D.N.; Yang, H.H.; Li, M.H.; Shi, H.J.; Zhang, X.B.; Wang, D.S. gsdf is a downstream gene of $d m r t 1$ that functions in the male sex determination pathway of the nile tilapia. Mol. Reprod. Dev. 2016, 83, 497-508. [CrossRef] [PubMed]

43. Zhang, X.; Guan, G.J.; Li, M.Y.; Zhu, F.; Hong, Y.H. Autosomal gsdf acts as a male sex initiator in the fish medaka. Sci. Rep. 2016, 6, 19738. [CrossRef] [PubMed]

44. Nakamura, S.; Watakabe, I.; Nishimura, T.; Toyoda, A.; Taniguchi, Y.; Tanaka, M. Analysis of medaka sox 9 orthologue reveals a conserved role in germ cell maintenance. PLOS ONE 2012, 7, 12. [CrossRef]

45. Yang, Y.; Wang, J.; Li, Y.; Zhou, Z.L.; Gui, J.F. Sequential, divergent, and cooperative requirements of foxl $2 a$ and foxl2b in ovary development and maintenance of zebrafish. Genetics 2017, 205, 1551-1572. [CrossRef] [PubMed]

46. Yu, H.Y.; Wang, Y.J.; Wang, M.Y.; Liu, Y.X.; Cheng, J.; Zhang, Q.Q. Growth differentiation factor 9 ( $g d f 9$ ) and bone morphogenetic protein 15 (bmp15) are potential intraovarian regulators of steroidogenesis in Japanese flounder (Paralichthys olivaceus). Gen. Comp. Endocrinol. 2020, 297, 113547. [CrossRef] [PubMed]

47. Dranow, D.B.; Hu, K.; Bird, A.M.; Lawry, S.T.; Adams, M.T.; Sanchez, A.; Amatruda, J.F.; Draper, B.W. Bmp15 is an oocyte-produced signal required for maintenance of the adult female sexual phenotype in zebrafish. PLoS Genet. 2016, 12, 24. [CrossRef] 
48. Wu, T.L.; Cheng, Y.Y.; Liu, Z.L.; Tao, W.J.; Zheng, S.Q.; Wang, D.S. Bioinformatic analyses of zona pellucida genes in vertebrates and their expression in Nile tilapia. Fish Physiol. Biochem. 2018, 44, 435-449. [CrossRef]

49. Dong, Z.D.; Zhang, N.; Liu, Y.; Xu, W.T.; Cui, Z.K.; Shao, C.W.; Chen, S.L. Expression analysis and characterization of zglp1 in the Chinese tongue sole (Cynoglossus semilaevis). Gene 2019, 683, 72-79. [CrossRef]

50. Miao, L.Y.; Yuan, Y.; Cheng, F.; Fang, J.S.; Zhou, F.; Ma, W.R.; Jiang, Y.; Huang, X.H.; Wang, Y.C.; Shan, L.J.; et al. Translation repression by maternal RNA binding protein Zar1 is essential for early oogenesis in zebrafish. Development 2017, 144, 128-138. [CrossRef]

51. Corina, H.; Walter, S.; Astrid, B. Genetics of sexual development: An evolutionary playground for fish. Genetics 2014, 196, 579-591. [CrossRef]

52. Stelkens, R.B.; Wedekind, C. Environmental sex reversal, Trojan sex genes, and sex ratio adjustment: Conditions and population consequences. Mol. Ecol. 2010, 19, 627-646. [CrossRef] [PubMed]

53. Yamamoto, Y.; Zhang, Y.; Sarida, M. Coexistence of genotypic and temperature-dependent sex determination in pejerrey Odontesthes bonariensis. PLoS ONE 2014, 9, e102574. [CrossRef] [PubMed]

54. Sato, T.; Endo, T.; Yamahira, K.; Hamaguchi, S.; Sakaizumi, M. Induction of female-to-male sex reversal by high temperature treatment in medaka, Oryzias latipes. Zool. Sci. 2005, 22, 985-988. [CrossRef]

55. Shao, C.W.; Li, Q.Y.; Chen, S.L.; Zhang, P.; Lian, J.M.; Hu, Q.M.; Sun, B.; Jin, L.J.; Liu, S.S.; Wang, Z.J.; et al. Epigenetic modification and inheritance in sexual reversal of fish. Genome Res. 2014, 24, 604-615. [CrossRef]

56. Desprez, D.; Melard, C. Effect of ambient water temperature on sex determinism in the blue tilapia Oreochromis Aureus. Aquaculture 1998, 162, 79-84. [CrossRef]

57. Myosho, T.; Takehana, Y.; Hamaguchi, S.; Sakaizumi, M. Turnover of sex chromosomes in celebensis group medaka fishes. G3-Genes Genom. Genet. 2015, 5, 2685-2691. [CrossRef] [PubMed]

58. Takehana, Y.; Matsuda, M.; Myosho, T.; Suster, M.L.; Kawakami, K.; Shin-I, T.; Kohara, Y.; Kuroki, Y.; Toyoda, A.; Fujiyama, A.; et al. Co-option of Sox3 as the male-determining factor on the $\mathrm{Y}$ chromosome in the fish Oryzias dancena. Nat. Commun. 2014, 5, 4157. [CrossRef] [PubMed]

59. Herpin, A.; Braasch, I.; Kraeussling, M.; Schmidt, C.; Thoma, E.C.; Nakamura, S.; Tanaka, M.; Schartl, M. Transcriptional rewiring of the sex determining dmrt1 gene duplicate by transposable elements. PLoS Genet. 2010, 6, 15. [CrossRef]

60. Matsuda, M.; Sakaizumi, M. Evolution of the sex-determining gene in the teleostean genus Oryzias. Gen. Comp. Endocrinol. 2016, 239, 80-88. [CrossRef] [PubMed] 